\section{Adsorption of Linear and Branched Alkanes in the Zeolite Silicalite-1}

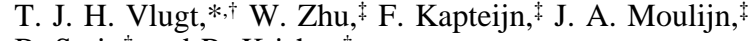 \\ B. Smit ${ }^{\dagger}$ and R. Krishna
}

Department of Chemical Engineering University of Amsterdam, Nieuwe Achtergracht 166 1018 WV Amsterdam, The Netherlands Department of Chemical Engineering Delft University of Technology, Julianalaan 136 2628 BL Delft, The Netherlands

Received December 22, 1997

Understanding the adsorption of hydrocarbons in zeolites is a prerequisite for optimizing catalytic processes using these materials. ${ }^{1,2}$ The focus of most experimental and theoretical studies has been to characterize the adsorption of linear hydrocarbons. ${ }^{3-6}$ Despite the importance of branched alkanes as lubricant or as high-octane-number fuel, our knowledge on the sorption of branched alkanes is far less. ${ }^{7,8}$ The adsorption behavior of normal alkanes in zeolites can be qualitatively different from that of branched alkanes. This can be demonstrated by comparison of the adsorption isotherms of $n$-butane and isobutane in silicalite- 1 (see Figure 1 (left)). While $n$-butane shows a monotonic approach to saturation, the corresponding isotherm for isobutane is nonmonotonic and exhibits an inflection point at a loading of 4 molecules per unit cell. Our objective is to provide a physical explanation for this remarkable observation.

To develop the explanation, we have performed molecular simulations in the Grand Canonical ensemble. ${ }^{9}$ In this ensemble, the zeolite is in contact with a reservoir that fixes the chemical potential and the temperature. In such a simulation, it is essential to successfully exchange particles with the reservoir. For small molecules, this can be achieved with conventional Monte Carlo techniques. To make these exchanges possible for long-chain alkanes, we used the configurational-bias Monte Carlo technique. ${ }^{10,11}$ With this technique, we grow a flexible alkane molecule atom by atom in such a way that the "empty spaces" in the zeolite are found. The bias of this growing scheme is removed exactly by a modification of the acceptance rules. ${ }^{11}$ The acceptance ratio of the particle exchange move is increased by 10 to 100 orders of magnitude and thus makes these simulations possible. ${ }^{6}$ In the simulations presented in this work, we used the

\footnotetext{
$\dagger$ University of Amsterdam.

* Delft University of Technology.

(1) Haag, W. O. In Zeolites and Related Microporous Materials: State of the Art 1994; Studies in Surface Science and Catalysis; Weitkamp, J., Karge, H. G., Pfeifer, H., Hölderich, W., Eds.; Elsevier: Amsterdam, 1994; Vol. 84 pp 1375-1394.

(2) Kärger, J.; Ruthven, D. M. Diffusion in Zeolites and other Microporous Solids; Wiley \& Sons: New York, 1992.
} 90.

(3) Stach, H.; Lohse, U.; Thamm, H.; Schirmer, W. Zeolites 1986, 6, 74-

(4) Abdul-Rehman, H. B.; Hasanain, M. A.; Loughlin, K. F. Ind. Eng. Chem. Res. 1990, 29, 1525-1535.

(5) Richard, R. E.; Rees, L. V. C. Langmuir 1987, 3, 335-340.

(6) Smit, B.; Maesen, T. L. M. Nature 1995, 374, 42-44.

(7) Cavalcante, C. L., Jr.; Ruthven, D. M. Ind. Eng. Chem. Rev. 1995, 34 $177-184$.

(8) June, R. L.; Bell, A. T.; Theodorou, D. N. J. Phys. Chem. 1990, 94, $1508-1516$

(9) Frenkel, D.; Smit, B. Understanding Molecular Simulations: from Algorithms to Applications; Academic Press: San Diego, CA, 1996.

(10) Frenkel, D.; Mooij, G. C. A. M.; Smit, B. J. Phys.: Condens. Matter 1992, 4, 3053-3076.

(11) Smit, B. Mol. Phys. 1995, 85, 153-172.
Table 1. Lennard-Jones Parameters Used in This Study

\begin{tabular}{lcc}
\hline & $\epsilon / k_{\mathrm{B}} /[\mathrm{K}]$ & $\sigma /[\AA]$ \\
\hline $\mathrm{CH}_{3}$ & 98.1 & 3.77 \\
$\mathrm{CH}_{2}$ & 47.0 & 3.93 \\
$\mathrm{CH}$ & 12.0 & 4.10 \\
$\mathrm{CH}_{3}-\mathrm{O}$ & 80.0 & 3.60 \\
$\mathrm{CH}_{2}-\mathrm{O}$ & 58.0 & 3.60 \\
$\mathrm{CH}-\mathrm{O}$ & 58.0 & 3.60 \\
\hline
\end{tabular}

${ }^{a}$ The parameters for the alkane-alkane interactions are from ref 12. The parameters for the alkane-zeolite interactions were fitted to reproduce the Henry coefficient and the heat of adsorption at zero coverage for various linear and branched alkanes. The combining rules of the Lennard-Jones parameters are $\epsilon_{i j}=\sqrt{\epsilon_{i i} \epsilon_{j j}}$ and $\sigma_{i j}=\sqrt{\sigma_{i i} \sigma_{j j}}$.

united atom representation for alkanes, in which the $\mathrm{CH}_{3}, \mathrm{CH}_{2}$, and $\mathrm{CH}$ groups are considered as pseudoatoms. ${ }^{12}$ Following Kiselev and co-workers, ${ }^{13}$ the zeolite is assumed to be rigid and the interactions of the alkane with the zeolite are dominated by the oxygen atoms of the zeolite. The alkane-zeolite and alkanealkane interactions are described by a Lennard-Jones potential with parameters shown in Table 1. The intramolecular interactions include bond-bending and torsion potentials and a fixed $\mathrm{C}-\mathrm{C}$ bond-length. ${ }^{12}$ Our simulation box consists of $16(2 \times 2 \times 4)$ unit cells of silicalite. A total simulation consisted of at least $10^{6}$ Monte Carlo steps.

In Figure 1 (left), the simulated adsorption isotherms are compared with the experimental ones. For both $n$-butane and isobutane the agreement between experiments and simulations is remarkably good. Furthermore, the simulations are able to reproduce the experimentally observed inflection point in the isotherm of isobutane.

Silicalite forms a three-dimensional network of straight and zigzag channels which cross at the intersections. Figure 2 shows the probability distribution of $n$-butane (left) and isobutane (middle) at low pressures. The differences are striking. Whereas $n$-butane has an equal probability to be in the straight channel, zigzag channel, or intersection, isobutane has a strong preference for the intersection. Let us now compare the siting of isobutane before (low loading, Figure 2 (middle)) and after (high loading, Figure 2 (right)) the inflection point in the isotherm. Below a loading of 4 molecules per unit cell, isobutane occupies only the intersections. At a loading of 4 molecules per unit cell, the intersections are fully occupied, and in order to achieve higher loadings, isobutane must also seek residence in the other channels. This, however, is energetically very demanding and requires a significantly higher driving force (pressure) resulting in the inflection point.

To obtain insights in chain length effects, we simulated the adsorption isotherms of 2-methylbutane, 2-methylpentane, and 2-methylhexane (Figure 1 right). The behavior of 2-methylbutane is similar to isobutane. For 2-methylpentane and 2-methylhexane, the inflection behavior is so pronounced that it results in a plateau. At low pressures, these branched alkanes are located at the intersections with their tails either in the straight channels or in the zigzag channels. At higher pressures, additional 2-methylpentane molecules can also be located in the zigzag channels (the straight channels are too short). This requires, however, that molecules in nearby intersections have their tails in the straight channels such that the zigzag channels are free. This additional entropy effect gives rise to the plateau for 2-methylpentane.

(12) Siepmann, J. I.; Martin, M. G.; Mundy, C. J.; Klein, M. L. Mol. Phys. 1997, 90, 687-693.

(13) Bezus, A. G.; Kiselev, A. V.; Lopatkin, A. A.; Du, P. Q. J. Chem Soc., Faraday Trans. 2 1978, 74, 367-379.

(14) Zhu, W.; van de Graaf, J. M.; van den Broeke, L. J. P.; Kapteijn, F.; Moulijn, J. A. Ind. Eng. Chem. Res., In press. 

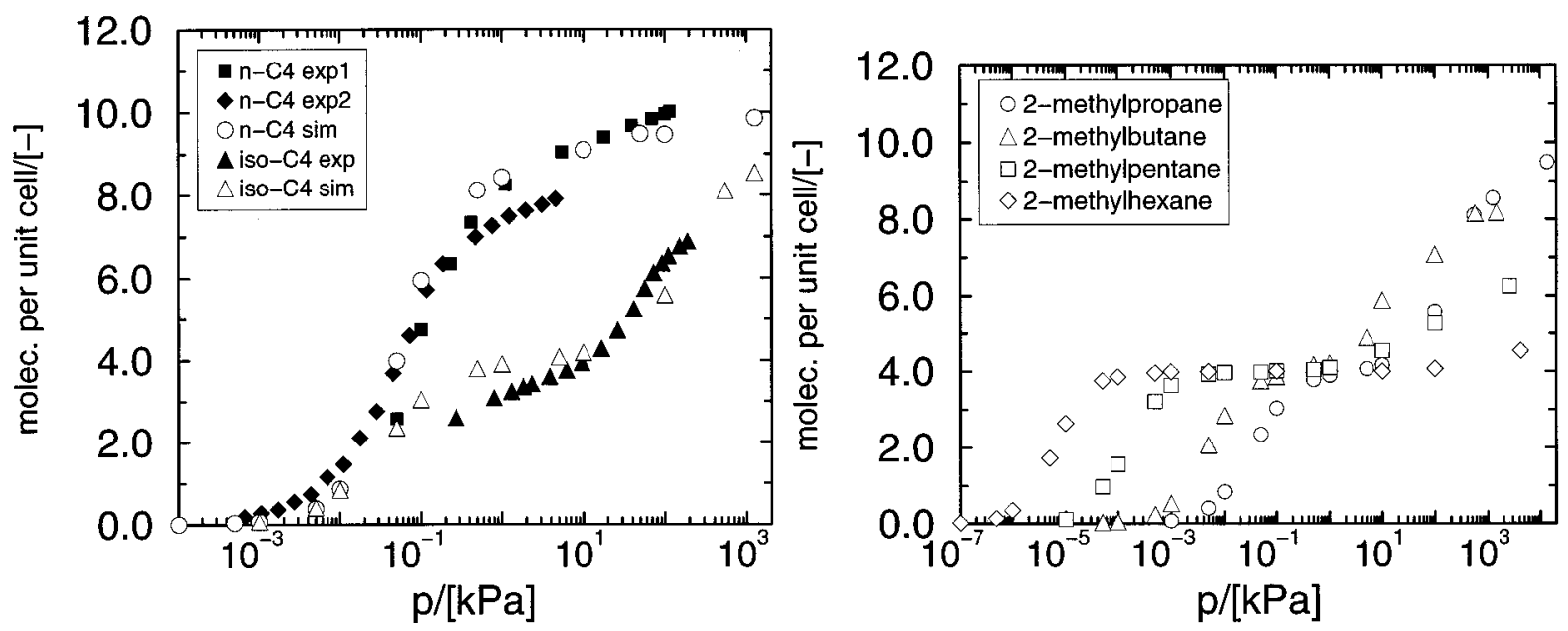

Figure 1. Adsorption isotherms of (left) $n$-butane and isobutane and (right) isobutane, 2-methylbutane, 2-methylpentane, and 2-methylhexane on silicalite-1 at $300 \mathrm{~K}$. The open symbols indicate simulations. The experimental data for $n$-butane was taken from ref 4 (exp1) and ref 5 (exp2), and the isotherm of isobutane was measured by us using a tapered element oscillating microbalance pulse mass analyzer. ${ }^{14}$ In the simulations, the chemical potential is used as input variable. For the conversion to pressures, we have used fugacity coefficients calculated from the Peng-Robinson equation of state.
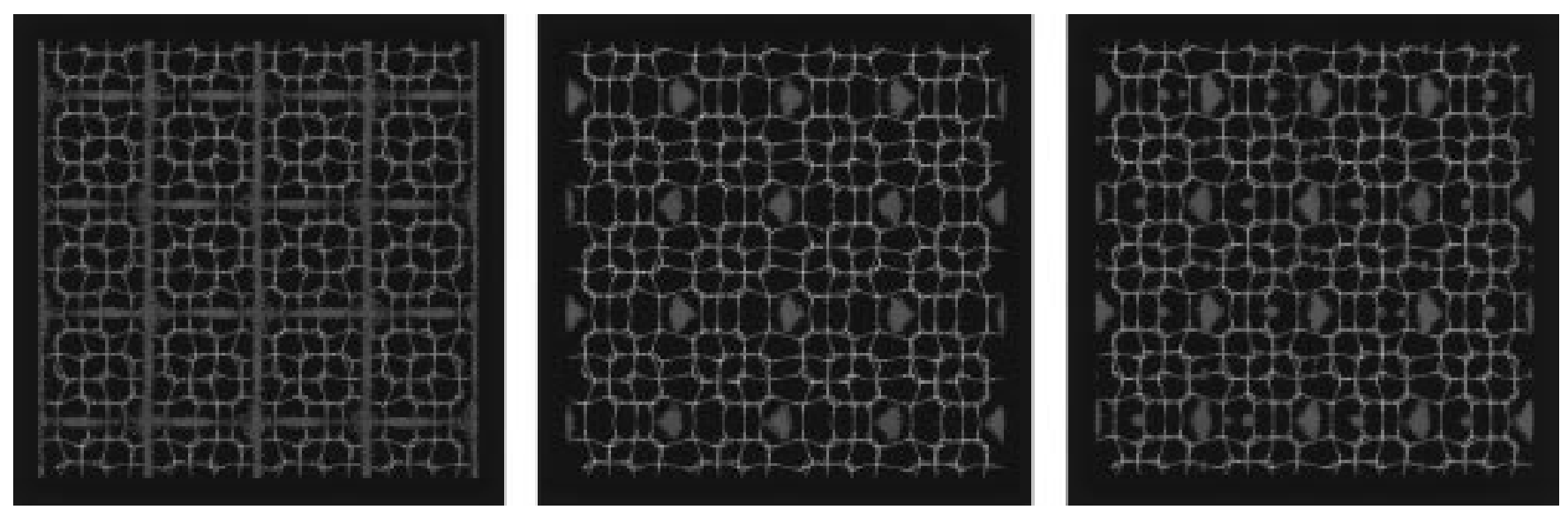

Figure 2. Probability distributions of $n$-butane at $0.1 \mathrm{kPa}$ (left), isobutane at $0.1 \mathrm{kPa}$ (middle), and isobutane at $100 \mathrm{kPa}$ (right) on silicalite-1 at 300 $\mathrm{K}$. The zigzag channels are from the left to the right, and the straight channels are perpendicular to the zigzag channels. These figures were obtained by plotting the centers of mass of the molecules (blue dots) every 500 Monte Carlo cycles. One periodic cell is depicted.

2-Methylhexane is so large that extreme pressures are required to squeeze molecules into the zigzag channels.

In this study, we pointed out a qualitative difference in the adsorption isotherms of normal and branched alkanes on silicalite1 , wherein the branched alkanes show inflection behavior. Since the adsorption isotherm has a significant influence on the concentration dependence of the diffusivity of molecules, ${ }^{2}$ we should expect curious behavior at the inflection point. This deserves further study and verification. A clear understanding of sorption and diffusion mechanisms, with help of molecular simulations, will lead to new and improved techniques for separation of alkanes from their isomers.

Acknowledgment. Financial support provided to T.J.H.V. from SON (Stichting Scheikundig Onderzoek Nederland) and to B.S. via the Graduate School on Process Technology (OSPT) are gratefully acknowledged. Part of the computer resources were generously provided by SARA (Stichting Academisch Rekencentrum Amsterdam)

JA974336T 